\title{
Supported cognitive-behavioural therapy self-help versus treatment-as-usual for depressed informal caregivers of stroke survivors (CEDArS): feasibility randomised controlled trial
}

\author{
Paul Farrand ${ }^{1}$, Joanne Woodford ${ }^{2} \star(1)$, Chelsea Coumoundouros ${ }^{2}$ and Frida Svedin ${ }^{2}$ \\ ${ }^{1}$ Clinical Psychology, Education, Development and Research (CEDAR), Psychology, University of Exeter, Perry Road, Devon \\ EX4 4QG, UK and 2Clinical Psychology in Healthcare, Department of Women's and Children's Health, Uppsala University, \\ 75123 Uppsala, Sweden \\ *Corresponding author. Email: joanne.woodford@kbh.uu.se
}

(Received 17 September 2019; revised 19 May 2020; accepted 20 May 2020)

\begin{abstract}
Demands placed on informal caregivers can result in an increased likelihood of experiencing common mental health difficulties that may affect their ability to undertake the caring role. Currently, however, few evidence-based interventions have been specifically developed for informal caregivers and available interventions are difficult to access. The Improving Access to Psychological Therapies (IAPT) programme aims to improve access to evidence-based psychological therapies for all groups and may therefore present an opportunity to meet informal caregiver needs. Located within the MRC Complex Intervention Framework, a Phase II feasibility randomised controlled trial (RCT) examines key methodological, procedural and clinical uncertainties associated with running a definitive Phase III RCT of an adapted written cognitive behavioural therapy (CBT) self-help intervention for informal caregivers of stroke survivors. Recruitment was low despite different recruitment strategies being adopted, highlighting significant challenges moving towards a Phase III RCT until resolved. Difficulties with study recruitment may reflect wider challenges engaging informal caregivers in psychological interventions and may have implications for IAPT services seeking to improve access for this group. Further attempts to develop a successful recruitment protocol to progress to a Phase III RCT examining effectiveness of the adapted CBT self-help intervention should be encouraged.
\end{abstract}

\section{Key learning aims}

After reading this article, readers should be able to:

(1) Consider key feasibility issues with regard to recruitment and attrition when running a randomised controlled trial of an adapted written cognitive behavioural therapy (CBT) self-help intervention for informal caregivers of stroke survivors.

(2) Understand potential barriers experienced by an informal caregiving population to accessing psychological interventions.

(3) Appreciate implications for clinical practice to enhance access to IAPT services and low-intensity CBT working with an informal caregiver population.

Keywords: CBT; cultural adaptation; depression; primary care; self-help

\footnotetext{
(c) British Association for Behavioural and Cognitive Psychotherapies 2020. This is an Open Access article, distributed under the terms of the Creative Commons Attribution licence (http://creativecommons.org/licenses/by/4.0/), which permits unrestricted re-use, distribution, and reproduction in any medium, provided the original work is properly cited.
} 


\section{Introduction}

Stroke commonly occurs in people from late middle age and represents a leading cause of mortality and disability worldwide (Johnson et al., 2019). Significant physical, emotional, cognitive and behavioural impairment associated with stroke places large demands on stroke survivors (Lui and Nguyen, 2018), alongside wider society and the economy (Dunbar et al., 2018; Johnson et al., 2019). Given these long-term impairments, support is often provided by informal caregivers in the home (Sumathipala et al., 2012). However, the provision of informal care is associated with a range of personal and societal costs. Costs include high caregiving burden (Oliva-Moreno et al., 2018; Pucciarelli et al., 2018), reductions in paid employment and associated financial difficulties (Andrew et al., 2015), social isolation (Lou et al., 2017), loss of self-identify (Knowles et al., 2016) and reduced engagement with valued activities (Jellema et al., 2019). Provision of informal care is also associated with greater risk of poor physical health (Hiel et al., 2015) and mental health (Haley et al., 2015) in informal caregivers themselves, with pooled prevalence rates of 40.2 and $21.4 \%$ found for depressive and anxiety-related symptoms, respectively (Loh et al., 2017). The need for evidence-based psychological interventions to support the long-term mental health needs of informal caregivers of stroke survivors is therefore justified. However, the long-term mental health needs of informal caregivers have been neglected (Silva et al., 2013; Simon et al., 2009).

Despite evidence-based psychological interventions for common mental health difficulties existing, there is a large psychological treatment gap globally for both depression (Thornicroft et al., 2017) and anxiety disorders (Alonso et al., 2018). Across Europe, less than 30\% of people with mental health difficulties access treatment (Wittchen et al., 2011). To address this treatment gap, the Improving Access to Psychological Therapies (IAPT) programme has been implemented across England [Clark, 2011; National Collaborating Centre for Mental Health (NCCMH), 2019]. In the IAPT programme, access for the treatment of mild-to-moderate common mental health difficulties is enhanced through the delivery of psychological therapies within a stepped-care service delivery model (van Straten et al., 2015). Informed by guidance from the National Institute for Health and Care Excellence (NICE, 2009, 2013), patients are initially assessed and receive low-intensity cognitive behavioural therapy (LICBT) at Step 2 of the stepped-care model (Clark, 2018), with those not recovering stepped-up to an evidencebased high-intensity intervention. LICBT intervention delivery is based on CBT self-help resources provided through a range of written materials or digital technologies (Farrand and Woodford, 2013). With guided LICBT, support is provided face-to-face or over the telephone by a psychological wellbeing practitioner (PWP) workforce (Clark, 2018), with a systematic review demonstrating higher effect sizes for supported rather than self-administered self-help (Farrand and Woodford, 2013).

Guided LICBT therefore represents a flexible way to deliver evidence-based psychological therapies, making it well suited to address barriers to treatment experienced by informal caregivers given demands of the caregiving role (Woodford et al., 2014; Woodford et al., 2018). Indeed, a review of interventions for informal caregivers of stroke survivors recommended telephone or internet-administered support to increase access for this population (Bakas et al., 2014). However, evidence indicates that adapting LICBT interventions to represent the needs of specific populations is required to improve acceptability and engagement with adaptations made to interventions for Armed Forces veterans (Farrand et al., 2019), people with dementia (Farrand et al., 2016) and parents of children treated for cancer (Wikman et al., 2018). Failing to adapt LICBT interventions for specific populations may also account for smaller effect sizes experienced by people with chronic health conditions compared with an adult mental health population (Beatty and Lambert, 2013; Farrand and Woodford, 2015), whereas tailoring psychological interventions for informal caregivers resulted in interventions being more effective than non-tailored interventions (Bakas et al., 2014). This is of particular importance 
given that few psychological therapies have been adapted to accommodate the increased demands placed on informal caregivers (Krevers and Öberg, 2011).

Following the Medical Research Council (MRC) complex interventions framework (Craig et al., 2008), a written CBT self-help intervention for depression in informal caregivers of stroke survivors was developed (Woodford et al., 2014; Woodford et al., 2018). The CBT self-help intervention was designed to be supported by PWPs for delivery within IAPT services (Clark, 2011; Clark, 2018). A Phase II feasibility randomised controlled trial (RCT; Eldridge et al., 2016) was conducted to examine methodological and procedural uncertainties to inform decisions to progress to a definitive Phase III trial (Thabane et al., 2010). Specific uncertainties include: (1) recruitment; (2) attrition; and (3) collection of outcome measurements as per those adopted within the IAPT programme (Clark, 2018).

\section{Method}

\section{Trial design}

This was a single-blind parallel group feasibility RCT (Craig et al., 2008), comparing supported written CBT self-help for depressed informal caregivers of stroke survivors with a treatment-asusual (TAU) control group. It was reported in line with the CONSORT extension for randomised pilot studies (Eldridge et al., 2016). The full study protocol has been published (Woodford et al., 2014) with no methodological or procedural changes made after trial commencement.

\section{Participants}

Eligible participants were: (a) self-identified informal caregivers of stroke survivors; (b) caring for a stroke survivor who had been at home for a minimum of 2 months following most recent stroke episode; (c) aged 16 years or over; (d) met criteria for major depressive disorder as determined by the Clinical Interview Schedule (CIS-R; Lewis et al., 1992); (e) scored between 10 and 22 on the PHQ-9; and (f) able to read English (see Appendix 1 in Supplementary material for baseline characteristics). Non-eligible participants were: (a) with a self-identified severe and enduring mental health difficulty; (b) currently misusing alcohol, prescribed medication or illegal drugs; (c) acutely suicidal; or (d) receiving another formal psychological therapy.

\section{Setting}

Participants were recruited via primary care services, specialist stroke healthcare settings and community organisations. The CBT self-help intervention was delivered by PWPs within Outlook Southwest and Steps2Wellbeing IAPT Services operating within Cornwall and Dorset in southwest England.

\section{Recruitment}

Informed by an effective strategy for the recruitment of informal caregivers of people with dementia (Whitebird et al., 2011), participants were recruited via the following routes.

\section{Primary care}

Practice staff searched General Practice electronic case records for stroke survivors and manually identified known informal caregivers. Study information packs were sent to all identified informal caregivers via the post with informal caregivers able to reply to the research team via reply slip or telephone. General practitioners (GPs) were also able to refer informal caregivers directly and study posters were displayed in General Practice reception areas. 


\section{Specialist stroke care settings}

Acute and community-based stroke clinical teams (e.g. acute stroke units, stroke rehabilitation units) approached informal caregivers within these settings and provided study information packs. Interested informal caregivers could either consent to the clinical team passing their contact details to the study team or reply to the research team directly using a reply slip or the telephone.

\section{Community outreach}

Study posters and flyers were displayed in community-based stroke and informal caregiver charities (e.g. Stroke Association) or within their newsletters and social media.

In each setting, potential participants were provided with a study information pack including an invitation letter, patient information sheet and anonymised reply slip to record reasons for non-participation.

\section{Procedure}

A researcher spoke to all interested informal caregivers, provided additional study information and gained verbal consent from potential participants agreeing to be telephone-screened. If eligible, full written consent was obtained with a full eligibility screen conducted over the telephone or face-to-face. Participants then completed clinical outcome measurements representing the baseline.

\section{Randomisation, allocation concealment and blinding}

A web-based external randomisation service located at the Peninsula Clinical Trials Unit was used to randomly allocate participants to either supported CBT self-help or treatment as usual. Minimisation was used to ensure balance across study sites, depression score at baseline and gender. To preserve blinding, allocation details were emailed to a researcher not otherwise associated with the study who informed participants of the study arm they were allocated. Allocation details were also emailed to the PWP the participant had been allocated to. A researcher remaining blind to group allocation collected and analysed all clinical and feasibility outcome data (Woodford et al., 2014).

\section{Interventions}

\section{Written CBT self-help}

Participants received an assessment session (35 minutes) with a PWP from a participating IAPT service in Cornwall or Dorset. All study PWPs had completed the IAPT national curriculum for the education of PWPs (Richards et al., 2011a,b) from an accredited training provider. A further a 1-day training session related to specific factors associated with the adapted CBT self-help intervention was delivered by study authors (P.F. and J.W.) and involved an informal caregiver of a stroke survivor alongside a stroke healthcare professional. Following the initial assessment session, the CBT self-help workbook was introduced (Woodford and Farrand, 2013). The workbook was developed specifically for informal caregivers of stroke survivors and was informed by previous qualitative research exploring the difficulties experienced by informal caregivers with elevated symptoms of depression and anxiety (Woodford et al., 2018). This workbook introduced three single stand LICBT interventions: behavioural activation, problem solving and goal setting (Bennett-Levy et al., 2010). The single LICBT intervention best suited to the main presenting difficulty was chosen collaboratively between the PWP and informal caregiver. At the end of treatment all participants received relapse 
prevention adapted for the population. During support sessions of between 25 and 35 minutes, informal caregivers were provided with the workbook alongside guidance concerning use between sessions. Dependent on informal caregiver preference, assessment and support sessions were provided face-to-face or over the telephone. Consistent with the IAPT programme, the number of support sessions was determined collaboratively during case-management supervision (Richards, 2010). Participants could receive up to 12 support sessions, which is higher than the number commonly received for adults within the IAPT programme (Clark, 2018; NHS Digital, 2019). Routine case-management and clinical skills supervision was supplemented by an additional monthly group clinical supervision session (45 minutes) specific to the caregiver intervention provided by a PWP clinical educator trained in the intervention.

\section{Control}

This was TAU where participants received usual care, for example support provided by a GP or other healthcare provider.

\section{Outcomes}

Consistent with the aims of a feasibility RCT, primary outcomes associated with study recruitment and attrition were examined to inform the potential to progress to a definitive RCT (Craig et al., 2008; Eldridge et al., 2016). A priori progression criteria to inform whether or not to proceed to a future definitive trial were not set in the present study. Clinical outcome measurements were included to examine the feasibility of clinical outcomes data collection, including severity of depression (PHQ-9; Kroenke et al., 2001) and anxiety symptoms (GAD-7; Spitzer et al., 2006), functional impairment (WSAS; Mundt et al., 2002) and caregiver burden (CBS; Elmståhl et al., 1996). Additionally, the number, length and frequency of support sessions was recorded, alongside clinical outcomes per support session in accordance with the IAPT minimum data set (NCCMH, 2019). Additional clinical outcome measures addressing wider impacts were also collected (Appendix 2 in Supplementary material).

\section{Sample size}

As per standard practice in pilot RCTs, no formal power calculation was undertaken (Arain et al., 2010). The recommended sample size of 30 participants per arm for pilot RCTs was used (Browne, 1995), consistent with the median sample size found in pilot RCTs (Billingham et al., 2013).

\section{Statistical analysis}

Participant flow data were used to calculate recruitment and attrition rates. Odds ratios were calculated with $95 \%$ confidence intervals to compare attrition rates between study arms. All analysis was undertaken per-protocol. Descriptive summaries (means and standard deviations) for the PHQ-9, GAD-7, WSAS and CBS clinical outcomes are reported at baseline, and 4- and 6-month follow-up. Means and standard deviations are also reported for the number, length and frequency of support sessions.

\section{Results}

\section{Participant flow}

Recruitment flow into the study with reasons for exclusion at both initial and full screen are illustrated using a CONSORT diagram (Schulz et al., 2010; see Fig. 1). 


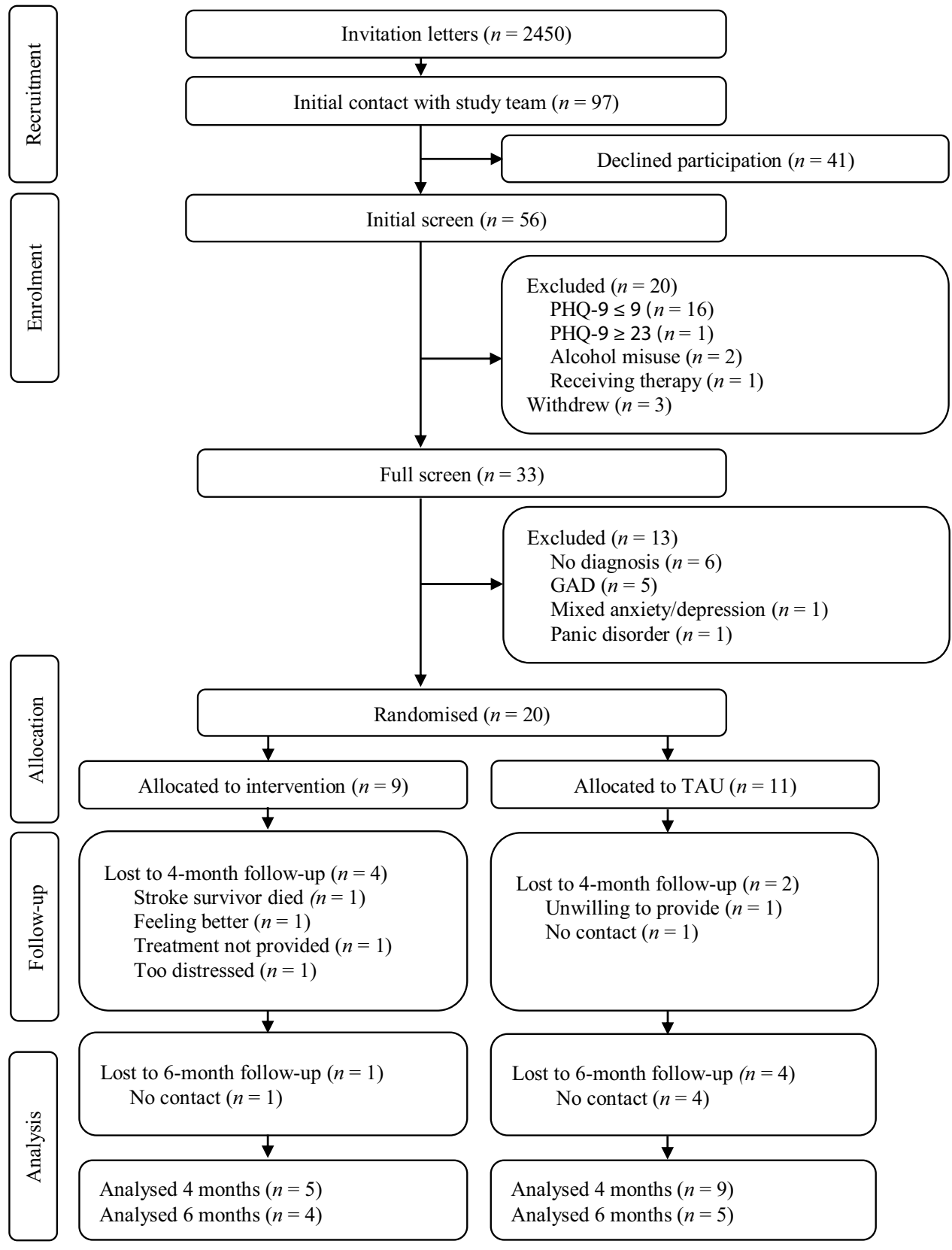

Figure 1. Consolidated Standards of Reporting Trials (CONSORT) flow diagram.

It cannot be known that community organisations distributed information packs to informal caregivers. Therefore, 284 information packs provided to community organisations are not included in the total number of invitation letters sent. Of the 2450 informal caregivers invited, $1 \%(20 / 2450)$ were randomised, with nine allocated to the intervention and 11 to the control (TAU). In total, $30 \%$ of participants (6/20) dropped out before 4-month post-randomisation follow-up (control: 2/11, 18\%; intervention: 4/9,44\%). An odds ratio of 3.6 [ $95 \%$ confidence interval (CI): 0.48-27.11] indicated no difference in drop-out at 4-month follow-up. 
Table 1. Reasons for non-participation

\begin{tabular}{|c|c|c|}
\hline Area & Reasons reported & $n(\%)$ \\
\hline Coping well & $\begin{array}{l}\text { (a) Not experiencing low mood or depression }(n=123) \\
\text { (b) Stroke survivor required little support }(n=86) \\
\text { (c) Coping well with the caregiving role }(n=55)\end{array}$ & $303(55)$ \\
\hline Barriers accessing support & $\begin{array}{l}\text { (a) Lack of time }(n=33) \\
\text { (b) Own poor physical health }(n=27) \\
\text { (c) Consider depression unrelated to the caregiving role }(n=14) \\
\text { (d) Not identifying as an informal caregiver }(n=12) \\
\text { (e) Consider main difficulty to be a lack of social care and practical } \\
\text { support }(n=11)\end{array}$ & $183(33)$ \\
\hline Procedural & $\begin{array}{l}\text { Mainly related to study procedural issues, including: } \\
\text { (a) Response received from the stroke survivor not caregiver }(n=16) \\
\text { (b) Invitation incorrectly sent, e.g. wrong address }(n=15) \\
\text { (c) Stroke survivor deceased }(n=4)\end{array}$ & $44(8)$ \\
\hline No point in the research & Unable to see any benefit to themselves from study participation & $3(0.5)$ \\
\hline No reason provided & Reason for non-participation form returned without a reason stated & $22(4.0)$ \\
\hline
\end{tabular}

Most frequently reasons provided only, therefore sample size $(n)$ reported under reasons reported does not equal total sample size for each area.

At 6-month follow-up, a total of 55\% participants (11/20) had dropped out of the study (control: 6/11, 55\%; intervention: 5/9, 56\%). An odds ratio of 1.04 (95\% CI: $0.18-6.12$ ) indicated no difference in drop-out at 6-month follow-up. Study arm status was disclosed to the researcher responsible for collecting data for $30 \%(6 / 11)$ of participants (disclosure from participant: 4/6, 67\%; disclosure from PWP: 2/6, 33\%).

\section{Feasibility of recruitment}

Randomisation rates for each source of recruitment were similar (General Practice: 15/2055, 0.7\%; stroke healthcare settings: $2 / 395,0.5 \%$; community outreach: $3 / 284,1 \%$ ). A detailed breakdown of enrolment stage by source of recruitment is presented in Appendix 3 in Supplementary material.

\section{Reasons for non-participation}

Of the 2450 invitation packs distributed, 376 (15\%) participants returned reasons for nonparticipation slip. In total, 555 individual reasons for non-participation were provided and categorised into four main areas (Table 1).

\section{Participant characteristics}

A summary of sociodemographic characteristics of informal caregiver participants and stroke survivors is presented in Appendix 1 in Supplementary material. Participants were predominantly female $(n=13,65 \%)$; White British $(n=19,95 \%)$ and the spouse or partner of the stroke survivor $(n=17,85 \%)$. Mean age of participants was 63 years $(S D=10.3)$ and had been in the caregiving role for a mean of 8 years $(S D=9.3)$.

\section{Clinical outcomes}

Means and standard deviations for each of the main clinical outcomes (PHQ-9, GAD-7, WSAS and CBS) can be seen in Appendix 4 in Supplementary material. Means and standard deviations for additional clinical outcomes were also collected (Appendix 5 in Supplementary material). Given the small sample size, participant drop-out being different between trial arms, and the 
Table 2. Variability in number, length and frequency of support sessions and individual session data ${ }^{a}$

\begin{tabular}{|c|c|c|c|c|c|c|}
\hline \multicolumn{7}{|l|}{ Session characteristics } \\
\hline & & \multicolumn{2}{|c|}{ Mean } & \multicolumn{2}{|l|}{$S D$} & Range \\
\hline Number of treatment sessions & & \multicolumn{2}{|c|}{4.0} & \multicolumn{2}{|l|}{1.4} & $3.0-6.0$ \\
\hline Number of do not attends & & \multicolumn{2}{|c|}{0.5} & \multicolumn{2}{|l|}{1.0} & $0.0-2.0$ \\
\hline Total duration of treatment (we & & \multicolumn{2}{|c|}{13.0} & \multicolumn{2}{|l|}{5.9} & $8.0-21.3$ \\
\hline Average length of treatment se & ons (min) & \multicolumn{2}{|c|}{30.8} & \multicolumn{2}{|l|}{1.0} & $30.0-32.0$ \\
\hline \multicolumn{7}{|l|}{ Clinical outcome measures } \\
\hline & \multicolumn{2}{|c|}{ PHQ-9 } & \multicolumn{2}{|c|}{ GAD-7 } & \multicolumn{2}{|c|}{ WSAS } \\
\hline & Mean $(S D)$ & Range & Mean $(S D)$ & Range & Mean $(S D)$ & Range \\
\hline Baseline & $15.0(4.2)$ & $11.0-20.0$ & $10.5(3.9)$ & $6.0-15.0$ & $22.3(10.3)$ & $13.0-35.0$ \\
\hline Initial assessment session & $16.0(8.3)$ & $7.0-27.0$ & $12.5(5.2)$ & $7.0-19.0$ & $26.3(7.9)$ & $16.0-35.0$ \\
\hline Final treatment session & $10.3(8.2)$ & $4.0-24.0$ & $7.0(7.0)$ & $2.0-19.0$ & $21.0(6.3)$ & $16.0-29.0$ \\
\hline 4-months post-randomisation & $6.3(3.2)$ & $4.0-11.0$ & $3.0(1.8)$ & $1.0-5.0$ & $14.4(14.5)$ & $0.0-27.5$ \\
\hline 6-months post-randomisation & $5.8(6.5)$ & $0.0-15.0$ & $4.5(5.9)$ & $0.0-13.0$ & $15.3(11.0)$ & $6.3-30.0$ \\
\hline
\end{tabular}

Means, standard deviations (SD) and ranges reported to 1 decimal place. PHQ-9, Patient Health Questionnaire-9; GAD-7, Generalised Anxiety Disorder 7-item Scale; WSAS, Work and Social Adjustment Scale. ${ }^{\text {DData a }}$ are presented for four of the five participants who remained in the study at 4-months post-randomisation. Session data were not provided by the PWP of one of the five participants, therefore their data is not included in this table.

aims of a feasibility study, these results are presented for descriptive purposes only and should be interpreted with caution.

\section{Variability in number, length and overall duration of support sessions and individual session data}

Of the five participants in the intervention arm who remained in the study at 4-months postrandomisation, four had complete data on support sessions and the main clinical outcomes. Number, length and overall duration of assessment and support sessions alongside baseline, initial assessment, final treatment and follow-up data for the PHQ-9, GAD-7 and WSAS is presented for the four participants with complete follow-up data (Table 2). Participants received a mean number of $4(S D=1.4$, range $=3-6)$ assessment and support sessions with mean session length of 30.8 minutes $(S D=1.0$, range $=30-32)$. The mean overall duration of treatment was 13 weeks $(S D=5.9$, range $=8.0-21.3)$. Investigation of the wide range for overall duration of treatment by the study team identified that PWPs were offering support sessions on a fortnightly basis, and in one case, the informal caregiver and PWP had difficulties in arranging support sessions around the informal caregiver's busy schedule.

At the initial assessment session, the mean PHQ-9 score was $16.0(S D=8.3$, range 7.0-27.0). Scores indicating severe depression on the PHQ-9 were reported by one participant, accounting for the large $S D$ and wide range in scores (Table 2).

The mean GAD-7 score at initial assessment was $12.5(S D=5.2$, range $=7.0-19.0)$. Again, scores indicating severe anxiety on the GAD-7 were reported by one participant, accounting for the large $S D$ and wide range in scores. By the final treatment session, the mean PHQ-9 score was $10.3(S D=9.5$, range $=4.0-24.0)$, slightly above caseness as defined as a score of $\leq 9$. The mean GAD-7 score was $7.0(S D=7.0$, range $=2-19)$, which was at caseness as defined by a GAD-7 score of $\leq 7$.

\section{Discussion}

Located within Phase II of the MRC Complex Interventions Framework (Craig et al., 2008), this study examined the feasibility of conducting an RCT comparing an adapted CBT self-help 
intervention for depressed informal caregivers of stroke survivors with treatment as usual. Results highlight significant challenges recruiting informal caregivers that need to be addressed prior to moving to a definitive Phase III (effectiveness) RCT. Given the feasibility study design, small sample size and participant drop-outs, clinical outcomes reported are solely for descriptive purposes and should be interpreted with caution.

Despite adopting multiple strategies to recruit informal caregivers into the present study, recruitment rates were low in all settings (General Practice, stroke specialist healthcare and community outreach). Although difficulties recruiting into depression trials are well documented (Hughes-Morley et al., 2015), the recruitment rates experienced in the present study are significantly lower than those found in depression trials conducted with a general mental health population (Richards et al., 2009; Richards et al., 2013). Systematic reviews examining interventions for informal caregivers have also highlighted small sample sizes to be common (Bakas et al., 2014; Brereton et al., 2007; Corry et al., 2015).

Barriers to informal caregivers accessing support potentially explain the poor recruitment rates experienced in this present study (Cameron et al., 2013; Denham et al., 2019a; Denham et al., 2019b). Consistent with barriers to accessing psychological treatment experienced by other populations, informal caregivers view mental health services as predominantly needed for severe psychological difficulties and perceive stigma seeking help and voicing problems (Mosher et al., 2015). Informal caregivers may also view their own needs as secondary to the needs of the person they are caring for and prioritise their caring role over self-care (Carduff et al., 2014; Greenwood and Smith, 2015; Mosher et al., 2015), identifying themselves in terms of the relationship to the person they are caring for rather than as a carer (Carduff et al., 2014; Greenwood et al., 2011). Informal care networks may also be large, with individuals having multiple caregivers (Burns et al., 2013; Koehly et al., 2015). Recruitment focused on the primary caregiver or closest next of kin may therefore miss the opportunity to recruit other network members who provide care.

Given that informal caregivers are also more likely to be late middle-aged and over, it may also be helpful to consider wider barriers to accessing mental health services experienced by an older population (Department of Health, 2009). Evidence suggests that stigma may be a particular challenge in terms of mental health help-seeking among older adults (Hall and Reynolds, 2014; Park and Unützer, 2011) with some older adults considering accessing treatment for depression as a weakness (van der Weele et al., 2012). Additionally, older adults have been found to view depression as an inevitable consequence of ageing, which may also present a barrier to accessing psychological treatment (Law et al., 2010). These attitudes resonate with those held by GPs that may view depression as an inevitable consequence of ageing (Collins and Corna, 2018). It is unsurprising therefore that GPs may normalise the experience of depression as a consequence of the caregiving role and fail to refer older caregivers into IAPT services.

To help address this barrier, the ability to self-refer has been adopted by the IAPT programme (NCCMH, 2019). This approach has been successful in engaging other groups representing diversity, for example in the Newham IAPT pilot site increasing access rates to reflect the ethnic mix of the population previously under-represented by GP referral alone (Clark, 2018).

Further efforts to close the psychological treatment gap (Rebello et al., 2014) have recommended that IAPT services develop closer links with community, third sector and charitable organisations that commonly engage under-represented populations (NCCMH, 2019). Several of these recommendations have been tailored specifically for informal caregivers by the Positive Step IAPT service (North Somerset) with treatment delivery adapted to address common barriers to access (National Health Service England, 2019). This approach to engagement is consistent with previous informal caregiver psychological intervention studies that have recruited successfully by utilising more assertive and collaborative community engagement approaches (Whitebird et al., 2011). IAPT services and future studies may 
therefore enhance recruitment rates by engaging more closely with informal caregiver focused community and charitable organisations.

A further important consideration was raised by the severe depression and anxiety scores reported by one informal caregiver at initial assessment and the final treatment session. Consistent with IAPT protocols, a function of weekly case management supervision is to monitor and support decision making regarding high scores and suicidality (Richards, 2010). However, to maintain blinding, individual session data were not provided to the trial team until the end of the trial. This presents an important ethical consideration concerning the design and conduct of pragmatic RCTs carried out in real-world clinical settings. A known difficulty concerning the design and conduct of research in real-world clinical settings is the blurring of boundaries between clinical care and research with respect to role delegation and responsibilities (Nicholls et al., 2019). Within this study, concerns associated with appropriate clinical decision-making for patients reporting consistently high outcomes may have been addressed by establishing an independent Data Monitoring and Ethics Committee to monitor clinical decisions being made. However, there is currently little guidance concerning the ethical design and conduct of pragmatic RCTs (Taljaard et al., 2018).

\section{Conclusion}

Substantial feasibility problems highlighted by this study, especially regarding recruitment, indicate that progression to a definitive Phase III (effectiveness) RCT is not feasible at the present time (Craig et al., 2008). However, a greater recognition of barriers to accessing support experienced by informal caregivers alongside enhancing links with community and charitable organisations, may overcome difficulties with recruitment and inform a further Phase II feasibility RCT (Craig et al., 2008).

\section{Limitations}

Although the study collected written responses highlighting reasons for non-participation, a thorough qualitative investigation may provide a better understanding regarding the acceptability of the CBT self-help intervention alongside an appreciation regarding barriers to study participation, help-seeking behaviour and barriers to accessing support. Finally, whilst study inclusion criteria did not exclude informal caregivers with moderately-severe to severe depression, recommendations for LICBT is restricted to mild-to-moderate severity (NCCMH, 2019). Including participants with more severe levels of depression may therefore require additional consideration before reaching conclusions about implementing LICBT for informal caregivers of stroke survivors within Step 2 of a stepped care model (Clark, 2018).

Supplementary material. To view supplementary material for this article, please visit https://doi.org/10.1017/ S1754470X20000239

Acknowledgements. The uthors would like to thank Steps2Wellbeing and Outlook Southwest Improving Access to Psychological Therapy Services for supporting the study. Interventions across both sites were supported by trial PWPs: Sam Gerdes, Vania Goldberger-Holland, Angela Mason, Ceri Summers and Dudley Poole, with Kathryn Rayson acting as PWP clinical supervisor. Lorna Cook undertook randomisation to retain blinding. We are especially grateful to the three members of our Patient and Public Involvement Trial Steering Committee.

Financial support. This work was funded by the Dunhill Medical Trust (grant number: RTF43/1111) with research support provided by the Western Comprehensive Local Research Network, the Primary Care Research Network, and the Stroke Research Network.

Conflicts of interest. Paul Farrand, Joanne Woodford, Chelsea Coumoundouros and Frida Svedin have no conflicts of interest with respect to this publication. 
Ethical statements. Authors have abided by the Ethical Principles of Psychologists and Code of Conduct as set out by the APA. The trial was conducted in accordance with Helsinki Declaration to ensure the welfare and rights of the study participants (World Medical Association, 2013). Informed written consent was obtained from all study participants. All data were securely stored and anonymised. The study was ethically approved by National Research Ethics Committee South West for Cornwall and Plymouth (REC reference number: 13/SW/0018).

Key practice points

(1) Lack of time and poor physical health experienced by informal caregivers should be considered significant barriers to access.

(2) IAPT services should consider promoting telephone support for CBT self-help as a way to address barriers and improve access for informal caregivers.

(3) To enhance access, IAPT services should consider greater engagement with informal caregiver focused community and charitable organisations rather than the primary caregivers themselves.

(4) Given that informal caregivers are likely to be middle-aged and older, it may be helpful to consult the evidence base on overcoming barriers for older people.

\section{Further reading}

National Collaborating Centre for Mental Health (2019). The Improving Access to Psychological Therapies Manual. London, UK: NCCMH. Available online at: https:/www.england.nhs.uk/wp-content/uploads/2019/12/iapt-manual-helpfulresources-v2.pdf

Silva, A. L., Teixeira, H. J., Teixeira, M. J. C., \& Freitas, S. (2013). The needs of informal caregivers of elderly people living at home: an integrative review. Scandinavian Journal of Caring Sciences, 27, 792-803. doi: 10.1111/scs.12019

Thornicroft, G., \& Tansella, M. (2014). Community mental health care in the future: nine proposals. Journal of Nervous and Mental Disease, 202, 507-512. doi: 10.1097/NMD.0000000000000151

Woodford, J., Farrand, P., Watkins, E. R., Richards, D. A., \& Llewellyn, D. J. (2014). Supported cognitive-behavioural selfhelp versus treatment-as-usual for depressed informal carers of stroke survivors (CEDArS): study protocol for a feasibility randomised controlled trial. Trials, 15, 157. doi: 10.1186/1745-6215-15-157

\section{References}

Alonso, J., Liu, Z., Evans-Lacko, S., Sadikova, E., Sampson, N., Chatterji, S., Abdulmalik, J., ... \& WHO World Mental Health Survey Collaborators (2018). Treatment gap for anxiety disorders is global: results of the World Mental Health Surveys in 21 countries. Depression and Anxiety, 35, 195-208. https://doi.org/10.1002/da.22711

Andrew, N. E., Kilkenny, M. F., Naylor, R., Purvis, T., \& Cadilhac, D. A. (2015). The relationship between caregiver impacts and the unmet needs of survivors of stroke. Patient Preference and Adherence, 9, 1065. https://doi.org/10.2147/PPA.S85147

Arain, M., Campbell, M. J., Cooper, C. L., \& Lancaster, G. A. (2010). What is a pilot or feasibility study? A review of current practice and editorial policy. BMC Medical Research Methodology, 10, 67. https://doi.org/10.1186/1471-2288-10-67

Bakas, T., Clark, P. C., Kelly-Hayes, M., King, R. B., Lutz, B. J., \& Miller, E. L. (2014). Evidence for stroke family caregiver and dyad interventions: a statement for healthcare professionals from the American Heart Association and American Stroke Association. Stroke, 45, 2836-2852. https://doi.org/10.1161/STR.0000000000000033

Beatty, L., \& Lambert, S. (2013). A systematic review of internet-based self-help therapeutic interventions to improve distress and disease-control among adults with chronic health conditions. Clinical Psychology Review, 33, 609-622. https://doi.org/ 10.1016/j.cpr.2013.03.004

Bennett-Levy, J., Richards, D. A., \& Farrand, P. (2010). Low intensity CBT interventions: a revolution in mental health care. In J. Bennett-Levy, D. A. Richards., P. Farrand., H. Christensen., K.M. Griffiths., D.J. Kavanagh, ... C. Williams (eds), Oxford Guide to Low Intensity CBT Interventions (pp. 3-18). Oxford, UK: Oxford University Press. https://doi.org/10.1093/ med:psych/9780199590117.003.0001

Billingham, S. A. M., Whitehead, A. L, \& Julious, S. A. (2013). An audit of sample sizes for pilot and feasibility trials being undertaken in the United Kingdom registered in the United Kingdom clinical research network database. BMC Medical Research Methodology, 13, 104. https://doi.org/10.1186/1471-2288-13-104

Brereton, L., Carroll, C., \& Barnston, S. (2007). Interventions for adult family carers of people who have had a stroke: a systematic review. Clinical Rehabilitation, 21, 867-884. https://doi.org/10.1177/0269215507078313

Browne, R. H. (1995). On the use of a pilot sample for sample size determination. Statistics in Medicine, 14, 1933-1940. https://doi.org/10.1002/sim.4780141709 
Burns, C. M., Abernethy, A. P., Dal Grande, E., \& Currow, D. C. (2013). Uncovering an invisible network of direct caregivers at the end of life: a population study. Palliative Medicine, 27, 608-615. https://doi.org/10.1177/ 0269216313483664

Cameron, J. I., Naglie, G., Silver, F. L., \& Gignac, M. A. M. (2013). Disability and rehabilitation stroke family caregivers' support needs change across the care continuum: a qualitative study using the timing it right framework. Disability \& Rehabilitation, 35, 315-324. https://doi.org/10.3109/09638288.2012.691937

Carduff, E., Finucane, A., Kendall, M., Jarvis, A., Harrison, N., Greenacre, J., \& Murray, S. A. (2014). Understanding the barriers to identifying carers of people with advanced illness in primary care: triangulating three data sources. BMC Family Practice, 15, 48. https://doi.org/10.1186/1471-2296-15-48

Clark, D. M. (2011). Implementing NICE guidelines for the psychological treatment of depression and anxiety disorders: the IAPT experience. International Review of Psychiatry, 23, 318-327. https://doi.org/10.3109/09540261.2011.606803

Clark, D. M. (2018). Realizing the mass public benefit of evidence-based psychological therapies: the IAPT program. Annual Review of Clinical Psychology, 14. https://doi.org/10.1146/annurev-clinpsy-050817-084833

Collins, N., \& Corna, L. (2018). General practitioner referral of older patients to Improving Access to Psychological Therapies (IAPT): an exploratory qualitative study. British Journal of Psychiatry Bulletin, 42, 115-118. https://doi.org/10.1192/ bjb. 2018.10

Corry, M., While, A., Neenan, K., \& Smith, V. (2015). A systematic review of systematic reviews on interventions for caregivers of people with chronic conditions. Journal of Advanced Nursing, 71, 718-734. https://doi.org/10.1111/jan.12523

Craig, P., Dieppe, P., Macintyre, S., Michie, S., Nazareth, I., \& Petticrew, M. (2008). Developing and evaluating complex interventions: the new Medical Research Council guidance. BMJ, 337, a1655. https://doi.org/10.1136/bmj.a1655

Denham, A. M., Wynne, O., Baker, A., Spratt, N., Turner, A., Magin, P. . . \& Bonevski, B. (2019a). 'This is our life now. Our new normal': a qualitative study of the unmet needs of carers of stroke survivors. PLoS ONE, 14, e0216682. https://doi. org/10.1371/journal.pone.0216682

Denham, A. M., Wynne, O., Baker, A. L., Spratt, N. J., \& Bonevski, B. (2019b). The unmet needs of carers of stroke survivors: an evaluation of Google search results. Health Informatics Journal, 146045821985253. https://doi.org/ $10.1177 / 1460458219852530$

Department of Health (2009). Older Person Positive Practice Guide. London, UK: Department of Health.

Dunbar, S. B., Khavjou, O. A., Bakas, T., Hunt, G., Kirch, R. A., Leib, A. R., . . \& \& Whitsel, L. P. (2018). Projected costs of informal caregiving for cardiovascular disease: 2015 to 2035: a policy statement from the American Heart Association. Circulation, 137, e558-e577. https://doi.org/10.1161/CIR.0000000000000570

Eldridge, S. M., Chan, C. L., Campbell, M. J., Bond, C. M., Hopewell, S., Thabane, L., \& Lancaster, G. A. (2016). CONSORT 2010 statement: extension to randomised pilot and feasibility trials. Pilot and Feasibility Studies, 2, 64. https://doi.org/10.1136/bmj.i5239

Elmståhl, S., Malmberg, B., \& Annerstedt, L. (1996). Caregiver's burden of patients 3 years after stroke assessed by a novel caregiver burden scale. Archives of Physical Medicine and Rehabilitation, 77, 177-182. https://doi.org/10.1016/ S0003-9993(96)90164-1

Farrand, P., Mullan, E., Rayson, K., Engelbrecht, A., Mead, K., \& Greenberg, N. (2019). Adapting CBT to treat depression in Armed Forces Veterans: qualitative study. Behavioural and Cognitive Psychotherapy, 47, 530-540. https://doi.org/ $10.1017 /$ S1352465819000171

Farrand, P. \& Woodford, J. (2013). Impact of support on the effectiveness of written cognitive behavioural self-help: a systematic review and meta-analysis of randomised controlled trials. Clinical Psychology Review, 33, 182-195. https:// doi.org/10.1016/j.cpr.2012.11.001

Farrand, P., \& Woodford, J. (2015). Effectiveness of cognitive behavioural self-help for the treatment of depression and anxiety in people with long-term physical health conditions: a systematic review and meta-analysis of randomised controlled trials. Annals of Behavioral Medicine, 49, 579-593. https://doi.org/10.1007/s12160-015-9689-0

Farrand, P., Woodford, J., Llewellyn, D., Anderson, M., Venkatasubramanian, S., Ukoumunne, O. C., ... \& Dickens, C. (2016). Behavioural activation written self-help to improve mood, wellbeing and quality of life in people with dementia supported by informal carers (PROMOTE): a study protocol for a single-arm feasibility study. Pilot and Feasibility Studies, 2, 42. https://doi.org/10.1186/s40814-016-0083-x

Greenwood, N., \& Smith, R. (2015). Barriers and facilitators for male carers in accessing formal and informal support: a systematic review. Maturitas, 82, 162-169. https://doi.org/0.1016/j.maturitas.2015.07.013

Greenwood, N., Mackenzie, A., Harris, R., Fenton, W., \& Cloud, G. (2011). Perceptions of the role of general practice and practical support measures for carers of stroke survivors: a qualitative study. BMC Family Practice, 12, 57. https://doi.org/ 10.1186/1471-2296-12-57

Haley, W. E., Roth, D. L., Hovater, M., \& Clay, O. J. (2015). Long-term impact of stroke on family caregiver well-being: a population-based case-control study. Neurology, 84, 1323-1329. https://doi.org/10.1212/WNL.0000000000001418

Hall, C. A., \& Reynolds III, C. F. (2014). Late-life depression in the primary care setting: challenges, collaborative care, and prevention. Maturitas, 79, 147-152. https://doi.org/10.1016/j.maturitas.2014.05.026 
Hiel, L., Beenackers, M. A., Renders, C. M., Robroek, S. J., Burdorf, A., \& Croezen, S. (2015). Providing personal informal care to older European adults: should we care about the caregivers' health? Preventive Medicine, 70, 64-68. https://doi.org/ 10.1016/j.ypmed.2014.10.028

Hughes-Morley, A., Young, B., Waheed, W., Small, N., \& Bower, P. (2015). Factors affecting recruitment into depression trials: systematic review, meta-synthesis and conceptual framework. Journal of Affective Disorders, 172, 274-290. https:// doi.org/10.1016/J.JAD.2014.10.005

Jellema, S., Wijnen, M. A., Steultjens, E. M., Nijhuis-van der Sanden, M. W., \& van der Sande, R. (2019). Valued activities and informal caregiving in stroke: a scoping review. Disability and Rehabilitation, 41, 2223-2234. https://doi.org/10.1080/ 09638288.2018.1460625

Johnson, C. O., Nguyen, M., Roth, G. A., Nichols, E., Alam, T., Abate, D., . . \& Adebayo, O. M. (2019). Global, regional, and national burden of stroke, 1990-2016: a systematic analysis for the Global Burden of Disease Study 2016. The Lancet Neurology, 18, 439-458. https://doi.org/10.1016/S1474-4422(19)30034-1

Knowles, S., Combs, R., Kirk, S., Griffiths, M., Patel, N., \& Sanders, C. (2016). Hidden caring, hidden carers? Exploring the experience of carers for people with long-term conditions. Health \& Social Care in the Community, 24, 203-213. https://doi. org/10.1111/hsc.12207

Krevers, B., \& Öberg, B. (2011). Support/services and family carers of persons with stroke impairment: perceived importance and services received. Journal of Rehabilitation Medicine, 43, 204-209. https://doi.org/10.2340/16501977-0649

Kroenke, K., Spitzer, R. L., \& Williams, J. B. (2001). The PHQ-9: validity of a brief depression severity measure. Journal of General Internal Medicine, 16, 606-613. https://doi.org/10.1046/j.1525-1497.2001.016009606.x

Lewis, G., Pelosi, A. J., Araya, R., \& Dunn, G. (1992). Measuring psychiatric disorder in the community: a standardized assessment for use by lay interviewers. Psychological Medicine, 22, 465-486. https://doi.org/10.1017/S0033291700030415

Loh, A. Z., Tan, J. S., Zhang, M. W., \& Ho, R. C. (2017). The global prevalence of anxiety and depressive symptoms among caregivers of stroke survivors. Journal of the American Medical Directors Association, 18, 111-116. https://doi.org/10.1016/ j.jamda.2016.08.014

Koehly, L. M., Ashida, S., Schafer, E. J., \& Ludden, A. (2015). Caregiving networks-using a network approach to identify missed opportunities. Journal of Gerontology Series B: Psychological Sciences and Social Sciences, 70, 143-154. https:// doi.org/10.1093/geronb/gbu111

Law, J., Laidlaw, K., \& Peck, D. (2010). Is depression viewed as an inevitable consequence of age? The 'understandability phenomenon' in older people. Clinical Gerontologist: Journal of Aging and Mental Health, 33, 194-209. https://doi.org/ 10.1080/07317111003773627

Lou, S., Carstensen, K., Jørgensen, C. R., \& Nielsen, C. P. (2017). Stroke patients' and informal carers' experiences with life after stroke: an overview of qualitative systematic reviews. Disability and Rehabilitation, 39, 301-313. https://doi.org/ $10.3109 / 09638288.2016 .1140836$

Lui, S. K., \& Nguyen, M. H. (2018). Elderly stroke rehabilitation: overcoming the complications and its associated challenges. Current Gerontology and Geriatrics Research, 9853837. https://doi.org/10.1155/2018/9853837

Mosher, C. E., Given, B. A., \& Ostroff, J. S. (2015). Barriers to mental health service use among distressed family caregivers of lung cancer patients. European Journal of Cancer Care, 24, 50-59. https://doi.org/10.1111/ecc.12203

Mundt, J. C., Marks, I. M., Shear, M. K., \& Greist, J. M. (2002). The Work and Social Adjustment Scale: a simple measure of impairment in functioning. British Journal of Psychiatry, 180, 461-464. https://doi.org/10.1192/bjp.180.5.461

National Collaborating Centre for Mental Health (NCCMH) (2019). The Improving Access to Psychological Therapies Manual. Retrieved from: https://www.england.nhs.uk/wp-content/uploads/2019/02/improving-access-to-psychologicaltherapies-manual.pdf (accessed 11 September 2019).

National Health Service England (2019). Positive Step for Carers in North Somerset. Retrieved from: https://www.england. nhs.uk/mental-health/case-studies/positive-step/ (accessed 23 January 2019).

NHS Digital (2019). Psychological Therapies: Annual Report on the Use of IAPT Services - England 2018-19. Leeds, UK: Health and Social Care Information Centre.

NICE (2009). Depression: Treatment and Management of Depression in Adults. Retrieved from: https://www.nice.org.uk/ guidance/GID-CGWAVE0725/documents/short-version-of-draft-guideline (accessed 12 September 2019).

NICE (2013). Social Anxiety Disorder: Recognition, Assessment and Treatment. Retrieved from: https://www.nice.org.uk/ guidance/cg159/resources/social-anxiety-disorder-recognition-assessment-and-treatment-pdf-35109639699397 (accessed 12 September 2019).

Nicholls, S. G., Carroll, K., Zwarenstein, M., Brehaut, J. C., Weijer, C., Hey, S. P., . . \& Taljaard, M. (2019). The ethical challenges raised in the design and conduct of pragmatic trials: an interview study with key stakeholders. Trials, $20,765$. https://doi.org/10.1186/s13063-019-3899-x

Oliva-Moreno, J., Peña-Longobardo, L. M., Mar, J., Masjuan, J., Soulard, S., Gonzalez-Rojas, N., ... \& Quintana, M. (2018). Determinants of informal care, burden, and risk of burnout in caregivers of stroke survivors: the CONOCES Study. Stroke, 49, 140-146. https://doi.org/10.1161/STROKEAHA.117.017575

Pilgrim, D., \& Carey, T. (2012). Improving access to psychological therapies: an account of recent policy aspirations in the UK and Australia. Advances in Mental Health, 10, 117-126. https://doi.org/10.5172/jamh.2011.10.2.117 
Park, M., \& Unützer, J. (2011). Geriatric depression in primary care. Psychiatric Clinics of North America, 34, 469-487. https://doi.org/10.1016/j.psc.2011.02.009

Pucciarelli, G., Ausili, D., Galbussera, A. A., Rebora, P., Savini, S., Simeone, S., . . \& Vellone, E. (2018). Quality of life, anxiety, depression and burden among stroke caregivers: a longitudinal, observational multicentre study. Journal of Advanced Nursing, 74, 1875-1887. https://doi.org/10.1111/jan.13695

Rebello, T. J., Marques, A., Gureje, O., \& Pike, K. M. (2014). Innovative strategies for closing the mental health treatment gap globally. Current Opinion in Psychiatry, 27, 308-314. https://doi.org/10.1097/YCO.0000000000000068

Richards, D. A. (2010). Supervising low intensity workers in high volume clinical environments. In Bennett-Levy, J., Richards, D. A., Farrand, P., Christensen, H., Griffiths, K. M., Kavanagh, D. J., ... \& Williams, C. (2010). Oxford Guide to Low Intensity CBT Interventions, pp. 129-137. Oxford, UK: Oxford University Press.

Richards, D. A., Farrand, P., \& Chellingsworth, M. (2011a). National Curriculum for the Education of Psychological Wellbeing Practitioners (2nd edn). London, UK: Improving Access to Psychological Therapies.

Richards, D. A., Chellingsworth, M., Hope, R., Turpin, G., \& Whyte, M. (2011b). Reach Out: National Programme Supervisor Materials to Support the Delivery of Training for Psychological Wellbeing Practitioners. London, UK: Rethink.

Richards, D. A., Hill, J. J., Gask, L., Lovell, K., Chew-Graham, C., Bower, P., . . \& Bland, J. M. (2013). Clinical effectiveness of collaborative care for depression in UK primary care (CADET): Cluster randomised controlled trial. BMJ, 347, f4913. https://doi.org/10.3310/hta20140

Richards, D. A., Hughes-Morley, A., Hayes, R. A., Araya, R., Barkham, M., Bland, J. M., ... \& Pilling, S. (2009). Collaborative Depression Trial (CADET): multi-centre randomised controlled trial of collaborative care for depression: study protocol. BMC Health Services Research, 9, 188. https://doi.org/10.1186/1472-6963-9-188

Schulz, K. F., Altman, D. G., \& Moher, D. (2010). CONSORT 2010 statement: updated guidelines for reporting parallel group randomised trials. BMC Medicine, 8, 18. https://doi.org/10.1186/1741-7015-8-18

Silva, A. L., Teixeira, H. J., Teixeira, M. J. C., \& Freitas, S. (2013). The needs of informal caregivers of elderly people living at home: an integrative review. Scandinavian Journal of Caring Sciences, 27, 792-803. https://doi.org/10.1111/scs.12019

Simon, C., Kumar, S., \& Kendrick, T. (2009). Cohort study of informal carers of first-time stroke survivors: profile of health and social changes in the first year of caregiving. Social Science \& Medicine, 69, 404-410. https://doi.org/10.1016/ j.socscimed.2009.04.007

Spitzer, R. L., Kroenke, K., Williams, J. B., \& Löwe, B. (2006). A brief measure for assessing generalized anxiety disorder: the GAD-7. Archives of Internal Medicine, 166, 1092-1097. https://doi.org/10.1001/archinte.166.10.1092

Sumathipala, K., Radcliffe, E., Sadler, E., Wolfe, C. D., \& McKevitt, C. (2012). Identifying the long-term needs of stroke survivors using the International Classification of Functioning, Disability and Health. Chronic Illness, 8, 31-44. https:// doi.org/10.1177/1742395311423848

Taljaard, M., Weijer, C., Grimshaw, J. M., Ali, A., Brehaut, J. C., Campbell, M. K., ... \& Fergusson, D. A. (2018). Developing a framework for the ethical design and conduct of pragmatic trials in healthcare: a mixed methods research protocol. Trials, 19, 525. https://doi.org/10.1186/s13063-018-2895-X

Thabane, L., Ma, J., Chu, R., Cheng, J., Ismaila, A., Rios, L. P., . . \& Goldsmith, C. H. (2010). A tutorial on pilot studies: the what, why and how. BMC Medical Research Methodology, 10, 1. https://doi.org/10.1186/1471-2288-10-1

Thornicroft, G., Chatterji, S., Evans-Lacko, S., Gruber, M., Sampson, N., Aguilar-Gaxiola, S., Al-Hamzawi, A., ... \& Kessler, R. C. (2017). Undertreatment of people with major depressive disorder in 21 countries. British Journal of Psychiatry, 210, 119-124. https://doi.org/10.1192/bjp.bp.116.188078

van Straten, A., Hill, J., Richards, D. A., \& Cuijpers, P. (2015). Stepped care treatment delivery for depression: a systematic review and meta-analysis. Psychological Medicine, 45, 231-246. https://doi.org/10.1017/S0033291714000701

van der Weele, G. M., de Jong, R., de Waal, M. W., Spinhoven, P., Rooze, H. A., .. \& Van Der Mast, R. C. (2012). Response to an unsolicited intervention offer to persons aged 75 years after screening positive for depression symptoms: a qualitative study. International Psychogeriatrics, 24, 270-277. https://doi.org/10.1017/S1041610211001530

Whitebird, R. R., Kreitzer, M. J., Lewis, B. A., Hanson, L. R., Crain, A. L., Enstad, C. J., \& Mehta, A. (2011). Recruiting and retaining family caregivers to a randomised controlled trial on mindfulness-based stress reduction. Contemporary Clinical Trials, 32, 654-661. https://doi.org/10.1016/j.cct.2011.05.002

Wikman, A., Kukkola, L., Börjesson, H., Cernvall, M., Woodford, J., Grönqvist, H., \& von Essen, L. (2018). Development of an internet-administered cognitive behavior therapy program (ENGAGE) for parents of children previously treated for cancer: participatory action research approach. Journal of Medical Internet Research, 20, e133. https://doi.org/10.2196/jmir.9457

Wittchen, H. U., Jacobi, F., Rehm, J., Gustavsson, A., Svensson, M., Jönsson, B., . . \& Fratiglioni, L. (2011). The size and burden of mental disorders and other disorders of the brain in Europe 2010. European Neuropsychopharmacology, 21, 655-679. https://doi.org/10.1016/j.euroneuro.2011.07.018

Woodford, J., \& Farrand, P. (2013). Helping Relatives and Carers of Stroke Survivors Overcome Depression. Exeter, UK: Exeter University. Available at: https://cedar.exeter.ac.uk/iapt/iaptworkbooksandresources/]

Woodford, J., Farrand, P., Watkins, E. R., \& Llewellyn, D. J. (2018). 'I don't believe in leading a life of my own; I lead his life': a qualitative investigation of difficulties experienced by informal caregivers of stroke survivors experiencing depressive and anxious symptoms. Clinical Gerontologist, 41, 293-307. https://doi.org/10.1080/07317115.2017.1363104 
Woodford, J., Farrand, P., Watkins, E. R., Richards, D. A., \& Llewellyn, D. J. (2014). Supported cognitive-behavioural selfhelp versus treatment-as-usual for depressed informal carers of stroke survivors (CEDArS): study protocol for a feasibility randomised controlled trial. Trials, 15, 157. https://doi.org/10.1186/1745-6215-15-157

World Medical Association (2013). World medical association declaration of Helsinki: ethical principles for medical research involving human subjects. JAMA, 310, 2191-2194. https://doi.org/10.1001/jama.2013.281053

Cite this article: Farrand P, Woodford J, Coumoundouros C, and Svedin F. Supported cognitive-behavioural therapy self-help versus treatment-as-usual for depressed informal caregivers of stroke survivors (CEDArS): feasibility randomised controlled trial. The Cognitive Behaviour Therapist. https://doi.org/10.1017/S1754470X20000239 